\title{
(Re)construccion de una identidad negra en Venezuela
}

Alain Charier

\section{(2) OpenEdition}

Édition électronique

URL : http://journals.openedition.org/plc/635

DOI : $10.4000 /$ plc. 635

ISSN : 2117-5209

Éditeur

L'Harmattan

Édition imprimée

Date de publication : 1 janvier 1998

Pagination : 275-293

ISSN : 1279-8657

\section{Référence électronique}

Alain Charier, " $(R e)$ construccion de una identidad negra en Venezuela », Pouvoirs dans la Caraïbe [En ligne], 10 | 1998, mis en ligne le 14 mars 2011, consulté le 21 avril 2019. URL : http:// journals.openedition.org/plc/635; DOI : 10.4000/plc.635 


\title{
( $R e$ construccion de una identidad negra en Venezuela
}

\author{
Dr. Alain CHARIER \\ Chargé de recherche CRPLC/CNRS \\ Martinica/Francia \\ (Ponencia presentada en la XXII Conferencia \\ Anual de la Asociación de Estudios \\ del Caribe, Barranquilla), 26 - 30/05/1997)
}

\section{DEL FOLKLORE A LA CULTURA POPULAR}

En Venezuela, las culturas étnicas o regionales no pudieron participar plenamente en los proyectos modernizadores promovidos por las élites, pero padecieron de las consecuencias de los mismos. El mito del mestizaje y el de la democracia racial $^{1}$ se unieron para reducir la cultura popular, especialmente de origen afro, a un espacio folklórico. Además, las regiones de población negra, cercanas en su mayoría las grandes ciudades, han sufrido un violento proceso de éxodo rural que ha debilitado sus identidades.

Sin embargo, se ha podido observar a partir de 1945 un nuevo interés hacia las manifestaciones culturales negras y indígenas. Según G. Carrera-Damas ${ }^{2}$ esta nueva actitud fue motivada por el peligro que representaba para el dominio de la burguesía criolla la llegada de una poderosa inmigración europea (por otra parte favorecida por ella a fin de «blanquear» el país), las élites quisieron promover una nueva identidad nacional sin modificar por lo tanto la jerarquia social.

${ }^{1}$. Wright Winthrop, Café con leche : a brief louk at race relations in twentieth century, Venezuela, Austin, University of texas Press, 1992.

2. Carrera-Damas Germán, El dominador cautivo, Caracas, Ed. Grijalbo, 1988. 
Se trataba sin embargo de un enfoque paseísta que veía en estas culturas unas sobrevivencias o reliquias del pasado, cerradas a cualquier tipo de evolución socio-económica. No se tomó en cuenta las diversas estrategias que empezaron a poner en marcha estos sectores de la población para (re)construirse como actores sociales.

Esta visión folklorista, que todavía sobrevive en el seno de varias instituciones, luce como muy inadecuada en un país donde más del $85 \%$ de la población es urbana y en el cual la mayoria de las manifestaciones culturales populares se desarrollan en el ámbito de la ciudad ; tampoco toma en cuenta el hecho de que ninguna parte del territorio ha escapado a la destructuración promovida por los medios de comunicación, por el sistema escolar, por el turismo masivo, y más recientemente por las políticas neoliberales en general.

A partir de los años ochenta una nueva generación de investigadores (herederos de M. Acosta-Saignes ${ }^{3}$ emp ezó a preocuparse por establecer un vínculo entre el estudio de las culturas populares y la búsqueda de políticas innovadoras en este campo, reubicando así varios aportes-especificamente el negro-dentro de la dinámica histórica de la sociedad venezolana. El problema de la identidad surge de ahora en adelante con más fuerza ${ }^{4}$.

Los acontecimientos que van a transformar la vida política, social y económica del país a partir de 1983, y sobre todo de 1989, van a dinamizar también estas propuestas y darles más resonancia.

${ }^{3}$. Acosta-Saignes Miguel, Vida de los esclavos negros en Venezuela, Caracas, Ed. Vavell Hermanos, 1967.

4. Montañez Ligia, El racismo scutto en una sociedad no racista, Caracas, Ed. Tropykos, 1993. 


\section{UN NUEVO INTERES HACIA LO CULTURAL}

Dentro de una situación duradera de crisis se encuentran elementos estructurales y conyunturales. En el caso venezolano si lo conyuntural surgió en los ochenta, lo estructural era mucho más antiguo y se remontaba, tal vez, hasta los inicios de la colonización misma y a la construcción progresiva de una red de privilegios y de clientelismos. Sin embargo, el fín de muchas certidumbres fue una oportunidad inesperada de promover la cultura en el mismo momento que se manifestaba la existencia de una incipiente "nueva sociedad civil".

Se pudo observar entonces una gran creatividad en todos los campos artísticos y una creciente demanda de parte de un público en busca de fuertes señales. Se constató también un aumento sustencial de los subsidios atribuidos por parte del Conac (Consejo nacional de la cultura) y - por primerea vez en la historia venezolana - la cultura se divisó como un desafío político mayor.

Esta evolución tiene varias causas : la cultura apareció en primer lugar como siendo una compensación a ofrecer a una ciudadanía fuertemente golpeada por los fracasos económicos y sociales ; era una manera de dar una dimensión humana a los ajustes neoliberales que habían hecho más visibles las desigualdades que hasta el momento, el populismo había sabido ocultar. La cultura se impuso como un factor de cohesión social y nacional. En esta última perspectiva, favorecer aunque sea de forma muy limitada - las culturas regionales y étnicas minoritarias podía aparecer como el inicio de una integración de las mismas como componentes de pleno derecho de la identidad nacional.

Había también por el medio el beneficio que se podía esperar al nivel internacional, más que todo cuando se trata de las culturas negras, en el Caribe insular. La dimensión económica no puede tampoco ser dejada de lado, no solamemte al considerar la cultura y sus producciones como fuentes de recursos sino también como factor de mejoramiento de la imagen empresarial. De hecho se pudo constatar 
cierta transformación de la actitud del sector privado al respecto, un impulso se dió hacia el mecenato.

En el contexto que acabamos de describir, la cultura popular afro que se había beneficiado de cierta revalorización y del trabajo de varias organizaciones populares, va a convertirse en un nuevo centro de interés por parte de los poderes políticos locales, regionales y nacional.

\section{LA CULTURA POPULAR COMO RETO PARA LOS PODERES}

Resultaría imposible abordar en el espacio limitado de esta ponencia el conjunto de acciones llevadas a cabo por varias instancias en pro del desarrollo de la cultura afro-venezolana, sin embargo un breve recorrido nos permitirá poner de relieve el empuje determinante dado por la sociedad civil a este proceso.

A partir de 1990 el Conac vió aumentar sustancialmente su presupuesto y a pesar de que el porcentaje reservado a la cultura popular se quedó muy limitado (todavía lo es), este sector se benefició también con este crecimiento. Pero más que todo, fue al nivel de los métodos de intervención donde se pudo observar cierta evolución de las mentes y la voluntad de hacer progresar un partenariado entre el Estado y los varios actores culturales.

Los gobernadores por su parte, a raíz de su elección directa (1989) buscaron afianzar su base política a través del reconocimiento de las culturas locales y étnicas. Promovieron textos y decretos en favor de ellas, pero frecuentemente sin acordar los subsidios necesarios para permitir realizaciones concretas.

Los municipios también beneficiarios de la reforma, mantuvieron generalmente una postura más tímida y su acción fue de hecho más limitada : en el ámbito rural frecuentemente por falta de recursos o de preparación de los responsables. Pero en las grandes ciudades como Caracas, donde las comunidades son tal vez más organizadas, la cultura popular ha sido tomada en cuenta, 
considerándola como un remedio parcial a los problemas engendrados por la marginalidad y la fragmentación étnico-social del territorio urbano.

De hecho, se ha subrayado abundantemente la importancia para toda acción cultural de la existencia de una comunidad claramente identificada, teniendo como base un territorio bien delimitado y historicamente compartido. La apropiación del pasado por ella debe permitir de no caer en las trampas de la unidad abstracta o de la diversidad irracional $^{5}$. Esta búsqueda de auto-identificación ha sido la base del desarrollo del movimiento cultural dentro de las comuninades negras rurales. La necesidad de estructurar comunidades nuevas ha estimulado en los barrios urbanos la acción de la sociedad civil.

\section{BARLOVENTO : "LABORATORIO" DE LA RECONSTRUCCION IDENTITARIA}

La acción de las organizaciones populares en Barlovento ha sido fundamental y ha convertido esta región en un laboratorio cultural permitiendo así la removilización de una población fuertemente golpeada económica y culturalmemte. Surgió un nuevo tipo de política cultural abierta a la participación real de sectores importantes de esta comunidad y propicia a la toma de palabra por parte de sus miembros que habían sido por demasiado tiempo únicamente objetos del discurso académico.

Estas organizaciones han tratado de abrir para Barlovento y sus tradiciones un camino hacia la modernidad, una modernidad diferente de la modernización incontrolada que había sufrido hasta el momento.Gracias a su s oder de convocatoria y a la acumulacion de experiencias que había logrado durante los diez últimos años, la sociedad civil supo convertirse en un interlocutor indispensable de los diversos poderes instituciones.

5. Guadarrama P.G. Pereliguin N., Lo universal y lo específico en la cultura, Bogotá, Ed. Quijana, Caballero, 1988, p. 93. 
Barlovento está ubicado a cien kilómetros al este de Caracas y abarca una superficie de $4610 \mathrm{~km}^{2}$, su población alcanza en la actualidad los 200000 habitantes. Desde hace mucho tiempo esta región a sido considerada como la esencia misma de la presencia negra en Venezuela. Tardíamente explotada (al iniziarse el siglo XVIII) conoció su aúge económico finalizándose el périodo colonial con la producción del cacao, rubro principal de exportacióon de la provincia. Esta especialización condujó a la existencia de la más grande concentración de esclavos que hubo en el país y al hecho de que, todavía, Barlovento sigue siendo la mayor zona de poblamiento negro de Venezuela.

A partir de la abolición de la esclavitud en 1854, la región entró en decadencia al nivel económico, proceso que se aceleró durante el siglo XX debido a un éxodo masivo hacia la capital y las zonas petroleras. La apertura de carreteras acabó con el aislamiento secular de Barlovento, y un turismo descontrolado provocó una deculturación aparentemente irreversible. Sin embargo, la población no quedó deprovista de recursós frente a un cambio tan violento. Tal vez porque era la heredera de una fuerte tradición de resistencia forjada en el cimarronaje $\mathbf{y}$ de una capacidad organizacional que se había manifestado por ejemplo a través del catolicismo popular ${ }^{6}$ y en el marco de la defensa de sus tierras comunitarias. De hecho su cultura, sea en el campo de la oralidad, de la música o de la danza, quedó mucho más vigente que las de otras partes del país.

Ya en los años cuarenta, bajo la influencia del escritor J.-P. Sojo aparecieron asociaciones culturales de raíz folklórica, las cuales se mantuvieron hasta los setenta. A esta última época un militantismo étnico-cultural nuevo empezó a desarrollarse en Barlovento, fue un movimiento que reflejaba una profunda preocupación al respecto de la política del Conac, orientada en aquel entonces hacia una folklorización despreciativa de la cultura popular local a través de

${ }^{6}$. Chacón Alfredo, Curiepe, ensayo sobre la realización del sentido en la actividad máyicoreligiosa de un pueblo venezuelano, Caracas, Ed. UCV, 1977. 
espectáculos para turistas (desgraciadamente esta tendencia no ha desaparecido completamente $)^{7}$.

Paradójicamente, un proyecto educacional iniciado por el Estado el Pasin (Pensamiento, Acción social, Identidad Nacional) sirvió de base para la unificación de las energías locales, permitió la emergencia de un nuevo tipo de organizaciones populares en Barlovento y frendró asi el ocaso cultural que se vislumbraba.

Resulta importante subrayar el contenido de este proyecto y las razones de su fracaso porque las circunstancias de este permitieron la toma de conciencia por parte de los actores locales de la necesidad de tomar en mano propia su desarrollo cultural. La nueva visión del proceso histórico que conllevaba el Pasin, centrada en lo local, encontró una violenta oposición por parte de varios intelectuales influyentes y el gobierno siguiente, a quien le tocó poner en marcha la reforma se dejó llevar por ellos y la abandono en 1986. Sin embargo 1a semilla quedó y los líderes comunitarios - más específicamente los de Curiepe y San-José - quienes habían participado en la experiencia, lanzaron sus proyectos autónomos, teniendo como objetivo el conocimiento de la comunidad por sí misma, su acercamiento a su propia génesis histórica y su toma de conciencia relativa a sus lazos con el Caribe y Africat ${ }^{8}$.

Se trataba de una estrategia que tenía como meta, la elaboración de una identidad venezolana más auténtica, capaz de abrir el camino a nuevas prácticas democráticas. Así nació en 1986 como asociación civil el CIDOCUB : Centro de Investigación y Documentación de la Cultura Barloventeña. Esta organización tenía tres ejes de trabajo fundamentales : poner en marcha una investigación participativa, reunir una documentación accesible a la población barloventeña, promover una enseñanza adaptada a la vida de la gente. Se trataba de considerar la comunidad en su conjunto como algo más que un grupo sin cohesión y sin objetivo comun.

7. Mosonyi Esteban, Identidad y culturas populares, Caracas, Ed. La Enseñanza viva, 1982, pp. 353-355.

${ }^{8}$ Entrevista con Tomás Ponce, Presidente del Cidocub, Curiepe, 26/05/1991. 
Los miembros fundadores del Cidocub se apoyaron también sobre el aporte de los especialistas (historiadores, sociólogos, antropólogos) oriundos de la región o muy ligados a ella, relacionando sus conocimientos a la memoria colectiva de la población.

Tal proceso encontró un eco favorable dentro de la comunidad, y más que todo en los pueblos más amenazados por las mutaciones nacidas del turismo como lo son Curiepe, Higuerote, San-José y RioChico.

Existía también la inquietud de ver los resultados de las investigaciones ser leídos, discutidos y criticados por la gente. Lo que determinó la creación de los dos centros de documentación de Curiepe y de San-José.

A partir de 1987, el Cidocub replanteó el problema del contenido de los pogramas escolares y llevó a cabo una reflexión al respecto, la cual le permitió presentar en 1990 proposiciones concretas al nuevo gobernador por primera vez elegido por voto popular y recibir una acogida favorable de parte de la dirección regional de la cultura. Negociado con el Cidocub, el proyecto de enseñanza de la historia regional trataba de favorecer la toma de conciencia de los maestros (partiendo de sus vivencias) y de promover las aptitudes de los alumnos como investigadores de sus proprias comunidades, eso a fín de poder publicar manuales que reflejasen realidades locales ${ }^{9}$.

Si se acepta la afirmación según la cual la existencia de manifestaciones culturales propias de una comunidad es un signo evidente su capacidad de movilización y de liderazgo ${ }^{10}$, se puede sin lugar a dudas constatar cierta declinación del Barlovento que hay sin embargo, que relativizar. Las situaciones locales siguen siendo muy diversas : algunas comunidades como Curiepe defienden con fuerza sus tradiciones, otras como Caucagua y San-José esperan el impulso de

9. Entrevista con Pilarica de Romero, Directriz de la cultura del Estado Miranda, Los Teques, 24/04/1991.

${ }^{10}$. Gonzales-Ordosgoítti, E.A. Siete fiestas residenciales canaqueñas, Caracas, Ed. Fundarte, 1991, p. 101. 
las organizaciones de la sociedad civil, unas quedan sin organización alguna.

Un hecho importante resulta ser la aparición de nuevos intercambios que se dan a través de varios festivales (Voz negra, Percusión de San-José) que han relacionado los pueblos de Barlovento con otras comunidades afro-venezolanas, afro-americanas e incluso africanas. La multiplicación de esos eventos que ocurren fuera del calendario festivo y religioso tradicional, sus origenes diversos, relativizan profundamente el uso del término «tradicional» para definir las manifestaciones que promoven. Se observa claramente que la mayoría no podría desarrollarse sín la convergencia de los esfuerzos de la sociedad civil y de varias instituciones oficiales, asi se vislumbra el cuadro general de la evolución actual de la cultura popular.

Unas iniciativas en el campo musical nos van a dar excelentes ejemplos de este fenómeno. La música es uno de los elementos más identificatorios de Barlovento, especialmente por la energía que ha brindado siempre a la resistencia cultural, pero desgraciadamente, también por los estereotipos que ha promovido como el del "negro tomando ron y tocando tambor". Particularmente rica en su registro rítmico y en su conjunto instrumental de percusiones (mina curbata, culepuya), la música de Barlovento no se ha salvado de la deculturación acelerada que ha sufrido la región. La Escuela de percusión de San-José, fundada en 1984, ha querido dar una respuesta global a este desafío, buscando demostrar así que la música podía ser una solución - puntual por cierto - a los problemas nacidos de una difícil realidad social que se caracteriza por un importante analfabetismo y un desempleo creciente dentro de la población jóven, favoreciendo un aumento de la delincuencia.

La experiencia empezó con una sensibilización de la comunidad a través de sus organizaciones de base (juntas vecinales, sindicatos agrícolas), después de esta fase se realizó una selección de los niños más dotados y por fin se instaló una red de transporte para permitir a los niños acudir a las clases en el centro de ensenanza ubicado en el pueblo de San-José. 
E1 contenido pedagógico propuesto era progresivo y se acercaba a la cultura afro-venezolana a través de una visión amplia que abarcaba las culturas negras en su conjunto, los varios géneros musicales del Caribe y el conocimiento necesario del solfeo-oportunidad para la alfabetizacion $^{11}$.

Promovido por miembros del Cidocub trabajando también en el seno del Conac, la escuela recibió el apoyo de este organismo y subsidios de la Dirección regional de la cultura, pero el objetivo inicial de los fundadores de extender el proyecto a otras comunidades negras de Venezuela no pudo darse por falta de recursos y la experiencia se ha detenido en San-José. Esta situación ilustra perfectamente el hecho que las iniciativas culturales nacidas de la propia sociedad civil necesitan todavía el apoyo oficial para poder crecer.

Caucagua, población al margen occidental de Barlovento y que se encuentra más y más absorbida por Caracas nos da un ejemplo singular : animadores culturales ligados a las organizaciones populares trataron de llevar a cabo una una acción dentro de una alcadia ${ }^{12}$ y tuvieron que volver al trabajo dentro de la comunidad. Este intento de cultura municipal (1990-92) fracasó por falta de recursos, y más que todo, debido a la ausencia de interés real por parte del alcalde, actitud que se puede observar - fuera del périodo electoral - en la mayoría de los municipios de Barlovento y de otras regiones del país. En Caucagua la fundación Victor Sosa se ha de hecho, transformada en e1 eje central de la vida cultural, esta organización es integrada por un conjunto de grupos musicales de varios estilós : percusionistas tradicionales, tocadores de jazz, salseros, músicos clásicos interesados en los temas ligados al patrimonio regional. Uno de ellos, el grupo experimental Caucaucuar es dirigido por el antoguo responsable cultural de la alcaldía. Esta formación desarrola un repertorio que mezcla piezas trdicionales y composiciones proprias que reflejan los

11. Ponencia de Miguel Urbina, Director de la escuela de percusiones de San-José, Encuentro Didáctico de los musicos percusionistas, Caracas, 26/10/1993.

12. Entrevista con Carlos Martinisky, Director municipal de la cuttura, Caucagua, 26/04/1991. 
problemas actuales de la comunidad. La fundación conduce una política de relaciones públicas que tiene como meta el reconocimiento de la cultura barloventeña al nivel nacional (se hace muy presente en Caracas) e internacional. Su competencia al nivel de la enseñanza musical le ha permitido imponerse como responsable del nuevo centro cultural financiado conjuntamente por el Conac y la Dirrección regional de la cultura, se trata del primer instituto de este tipo en Barlovento.

Río-Chico ha sido la sede de una experiencia muy original que tiene como meta del renacimiento socio-cultural y ciudadano gracias a la lucha en favor del medio ambiente. Sus iniciadores apoyándose sobre el hecho se que e Barlovento, por causa del desarrollo turístico anárguigo de su litoral - completamente urbanizado - corría un riesgo ecológico mayor (destrucción de su espacio que encierra las bases mismas de su cultura) decidieron hacer público el debate sobre este tema tan importante.

En esta perspectiva crearon en 1984 la asociación «Represénta te-tú $»^{13}$. Este nombre indicaba claramente la voluntad de sus miembros de promover, más allà de la simple denunciación de la contaminación, una nueva toma de responsabilidad por parte de la comunidad para asumir su propio futuro. Las causas fundamentales del problema ecológico en Barlovento son por una parte el afán de lucro de los promotores, quienes no han vacilado por ejemplo en destruir con productos quimcos los manglares de la Laguna de Tacarigua teoricamente Parque nacional - provocando asi un daño irremediable a su comunidad de pescadores : y por otra parte la irresponsabilidad - a veces la corrupción - de los ediles, quienes pretenden promover el turismo masivo como el único camino hacia el empleo y sobre todo la modernidad. El argumento econónico en ausencia de una formación real de la población joven aparece muy ilusoria.

Además un turismo desligado de las realidades socioeconómicas locales, ejerce una fuerte presión sobre las tierras agrícolas

13. Entrevista con Carlos Rodriguez, Presidente de « Represéntate-tú », Rio-Chico, 19/05/1991. 
y conotribuye a transformar las manifestaciones culturales en mero espectáculo.

La acción de Represéntate-tú es múltiple pero siempre se apoya sobre la movilización y la participación de la comunidad : juicios en contra de las empresas contaminadoras, organización de una cooperativa de reciclaje de la basura, iniciacción de los campesinos a las técnicas de recuperación de los suelos, sensibilización de los turistas al necesario respeto a las costumbres autóctonas. La organización publicó un periódico y aprovecho la oportunidad que le proporcionaron las elecciones municipales para dar a conocer sus reinvendicaciones y poder así obligara las autoridades locales a dar una respuesta a los problemas que había planteado. Es asi como ha sido realizada finalmente la tan necesaria laguna de oxidación.

Este breve panorama no sería completo si no se tomara en cuenta la acción de la Unión de las mujeres negras fundada en 1984. Conscientes de la doble discriminación que padecen en Venezuela ${ }^{14}$, como negras y como mujeres, sus miembros provocarón una reflexión en los medios de comunicación al respecto, denunciando los estereótipos dominantes y organizaron talleres de auto-estima a fin de luchar en contra del endoracismo difundido desde la infancia.

Esta organización ha promovido campañas para realzar el papel histórico y social de las mujeres negras por ejemplo en Barlovento dónde constituyen $40 \%$ de los jefes de familia y cumplen este papel dentro de una situación económica difícil y sin aceso a la educación. Proyectos de artesanía y de turismo ambiental han sido desarrollados, iniciando respuestas concretas ${ }^{15}$.

${ }^{14}$. Situación de la mujer negra, Caracas, Ed. Cisfe, 1992, p. 184.

15 . Entrevista con Reina Arrapia, Presidenta de la Asociación de las Mujeres negras de Venezuela, Caracas, 19/03/1997. 


\section{SITUACIONES HETEROGENEAS DE LAS COMUNIDADES NEGRAS}

E1 nivel alcanzado en su trabajo de reconstrucción identitaria por cada comunidad afro-venezolana varía mucho y se encuentra generalmente vinculado con el desarrollo de la sociedad civil local y con la institucionalización de la cultura popular promovida por los poderes regionales.

En el municipio Veroes del Estado Yaracuy, un asentamiento negro antiguo ha visto su estructura agrícola y socio-cultural (culto a San-Juan) completamente negada a raíz de la pérdida de la tenencia de sus tierras a favor de la producción azucarera industrializada. Hubo que esperar los años ochenta ${ }^{16}$ para que un grupo de animadores exteriores (pero algunos oriundos del municipio) se encargaran de "recrear" las manifestaciones tradicionales. Más recientemente, la autonomización del municipio, la nueva actitud de la gobernación y el crecimiento de asociaciones autóctonas (relacionadas frecuentemente al sindicalismo agrícola ${ }^{17}$ parecen abrir el camino hacia una real toma de conciencia identitaria de la población.

En el extremo oriente del país, la región costera de Cariaco/Carupanor y Paria (Estado Sucre) ha siempre sido caracterizada por las relaciones muy estrechas que mantiene con el Caribe insular (más que todo Trinidad, pero también con Granada y en un pasado no tan lejano con las islas francófonas) y tiene por lo mismo una cultura singular. Actualmente una red integrada por investigadores $^{18}$, Ateneos ${ }^{19}$, Casas de la cultura $^{20}$ y fundaciones

\footnotetext{
16. Entrevista con la Pra-Lisbella Paéz, san Felipe, 05/12/1996.

17. Entrevista con David-Villegas, Pr. Fundación «La misma gente », El Chino, 30/03/1997.

18. Entrevista con Juan de Dios Diaz, Ir. Instit. Est. Etnicos de Paria, Carúpano, 20/11/1996.

${ }^{19}$. Entrevista con Merbys Rodriguez, Director del Ateneo, Guiria, 21/11/1996.

20 Entrevista con Josué Villaroel, Uir-Casa de la Cultura, Yaguaraparo, 09/04/1997.
} 
privadas $^{21}$ trata de rescatar y de promover - en todos sus aspectos - esta identidad original. Sin embargo la apertura económica (forajes petroleros) no deja de ser una ameneza para las comunidades negras de esta región en proceso de reafirmación.

A lo largo del Litoral central (Municipio Vargas del Districto Federal, Estado Aragua) se puede observar una movilización de los pueblos negros alrededor de proyectos micro-económicos ligados al turismo (Chichi Riviche, La Sabana) o a la pesca (Chuspa). Estas comunidades buscan alcanzar de esta mamera un desarrollo sustentable negociando, a veces directamente, con actores extranjeros (Comunidad europea).

En el sur del Lago de Maracaïbo (Estados Zulia y Mérida) una zona de poblamiento negro antiguo con tradiciones culturales muy fuertes (Culto a San-Benito) pero sumamente pobre y olvidada por Gobernadores lejanos (Maracaïbo, Mérida) trata de proyectarse gracias a su Ateneo (Bobures). Luchas por el liderazgo socio-cultural local han obstaculizado un real progreso en este sentido.

\section{LA IDENTIDAD NEGRA EN LA CIUDAD}

En las principales ciudades de Venezuela, y más que todo en Caracas, cualquier politica cultural tiene que afrentarse a una situación muy compleja. La capital ha crecido sin planificación y los 680000 habitantes de 1950 son hoy más de 4,5 millones el $20 \%$ de la población de la nación. Este crecimiento es más que todo el resultado de un importantísimo movimiento migratorio desde el campo ; varias regiones negras (Barlovento, Litoral central) están muy presentes dentro de este proceso.

21. Entrevista con S. Vicentelli, «Fondación Proyecto Paria », Carúpano, 10/04/1997. 
Estos últimos contingentes se han agrupado generalmente en las áreas marginales (ranchitos, urbanizaciones pobres), zonas que por mucho tiempo han sufrido un abandono completo al nivel de la acción cultural por parte de autoridades, negandoles cualquier necesidad al respecto $^{22}$.

La Vega es un barrio relativamente "antiguo", que ha empezado a constituirse hace más de treinta años con la instalación de estas poblaciones rurales, que se ha extendido - como la mayoria de las comunidades de este tipo - sobre las laderas de un cerro : los últimos integrantes tuvieron que radicarse cada vez más arriba. Hoy en día, su centro conocido como la parroquía San-Juan, tiene una apariencia realmente urbana.

La población es esencialmente venezolana y proviene de varias partes del interior del país : Oriente, Andes, Litoral del Districto federal, Barlovento. En La Vega ha ya nacido y crecido toda una generaci país $n$.

E1 barrides - con razón - considerado como muy peligroso, pero es también el lugar de implantación de una fuerte tradición organizativa al nivel popular, y cuando fue fundado el grupo Autóctono en 1980 existían ya una junta de vecinos, clubes deportivos, un grupo de teatro y un grupo de danza nacionalista.

Autóctono fue originalmente un grupo de música popular venezolana. Sus fundadores, después de haber llevado a cabo un trabajo de investigación en el interior del país, orientaron su acción hacia la permanencia de las tradiciones en el seno de esta pequeña sociedad de inmigrantes de identidad incierta y frágil frente a los efectos destructuradores del ambiente capitalino ${ }^{23}$. E1 repertorio del grupo ha sido predominantemente afro-venezolano; de hecho muchos integrantes - incluyendo su presidente - son negros, pero la razón principal radica en el poder de convocatoria y de proyección de este componente cultural, lo cúal ha permitido una integración de la

22. Zuluoga Pablo, «San Agustín, La otra cara», Criticarte, n ${ }^{\circ}$ 6, Caracas, 05/1992.

${ }^{23}$ Entrevista con William Ochoa, Presidente de «Autóctono », La Vega, Caracas, 24/06/1994. 
comunidad, agregando inclusive miembros herederos de tradiciones culturales distintas.

Este proceso se ha visto reforzado por el nacimiento y el desarrollo del culto a San-Juan en esta parroquia (este culto se ha extendido a múltiples áreas de Caracas en el transcurso de los últimos años) y por la organización anual del "Encuentro de los Santos de los Negros de Venezuela". Se trata de hecho de dos manifestaciones muy distintas en lo que se refiere a sus origenes.

La primera corresponde al surgimiento de una tradición rural en el ambiente urbano a raíz de una promesa personal que recibió apoyo y respuesta por parte de la comunidad, la segunda resultó de la voluntad de varios actores culturales (con el apoyo del Conac y de Fundarteorganismo cultural del municipio Libertador) de poner en escena de hacer encontrarse en un mismo lugar varias tradiciones ligádas al catolicismo popular afro-venezolano.

Sin embargo en ambos casos se puede observar el nacimiento de una integración (local o nacional) al interior de vivencia común a la vez tradicional y moderno, rural y urbano. Resulta fundamental igualmente el compromiso de la comunidad al nivel de la acogida del público y de los grupos : ha permitido es solamente ella que el éxito de estas manifestaciones son el resultado de la cohesión que la cultura afro - a través de los organizaciones populares - ha llevado al barrio de La Vega.

Un proyecto comparable con el de "Autóctono" en La Vega está empezando a ponerse en marcha a una escala mucho más grande en Caracas : se trata de el de ACU (Asociacion de Cultores Urbanos). Catia es en realidad una zona immensa y diversa, sin clara definición administrativa, que abarca toda la parte occidental del Municipio Libertador (Caracas misma) y cuenta casi con dos millones de habitantes (40\% de la población capitalina).

Catia es también una suma de los problemas urbanos en América latina : segregación espacial étnico-social, divorcio entre 
instituciones y prácticas culturales, dificultad para construir nuevas identidades colectivas ${ }^{24}$.

Promover una identidad para Catia, construir una ciudadanía de proximidad, mejorar la vida de la población son los objetivos de los miembros fundadores de $\mathrm{Acu}^{25}$ oriundos ellos mismos de las varias organizaciones populares locales. En este proceso el papel de las manifestaciones culturales de origen negra resulta muy importante, el poder de convocatoria que ellas tienen permite una movilización inicial de la gente ${ }^{26}$.

Por otra parte, Catia cuenta con varias comunidades de inmigrantes extranjeros, unas negras como la de los haïtianos (agrupada en el barrio Los Magallanes). Esta última, trata de conciliar su deseo de integración y su voluntad de reafirmación identitaria gracias a una nueva organización independiente del poder político de su país de origen ${ }^{27}$.

Las dos experiencias que acabamos de evocar son muy recientes, y es todavía demasiado temprano para hacer un balance. Muy diferente es el caso del barrio San-Millán de Puerto-Cabello.

Otra vez marginado, San-Millán se ha convertido, a raíz del largo trabajo de la asociación "Tambores de San-Millán"28, en el simbolo de esta ciudad caribeña y le ha permitido reanudar sus lazos con las islas neerlandófonas vecinas(Curacao, Aruba). Sin embargo la falta de relevo del liderazgo fundador y una organización insuficiente asombrecen el futuro de un proyecto hasta el momento exitoso.

24. T. Bolívar, J. Baldó, La cuestión de los barrios, Ed. Monte Avila, Caracas, 1995.

25 . Entrevista con Charley Nora, miembro fundador de ACU, Caracas, 17/03/1997 .

26. Entrevista con Ricardo Linares, director del grupo «Cañon», Caracas, 25/04/1997.

27. Entrevista con Fritz Saint-Louis, presidente de la asociación Haïtiana, Caracas, 25/04/1997.

28. Entrevista con Hernán Vilanueva, director de «Tambores de San-Millán », Puerto-Cabello, 04/12/1996. 


\section{UN CAMBIO DURADERO ?}

Como lo hemos dicto anteriormente 1as experiencias evocadas aquí se han desarrollado, en su mayoría, dentro de un período relativamente breve $\mathrm{y}$, a veces, dentro de comunidades muy específicas, por lo tanto no permiten sacar conclusiones a largo plazo.

Los logros pueden revertirse, por ejemplo en Barlovento entre 1993 y 1996 cuando la situación se había vuelto muy tensa a raíz de un cambio de color político de la Gobernación : la cultura negra y las organizaciones populares habían sido relegadas a un papel secundario $^{29}$. Sin embargo en esta oportunidad, la sociedad civil y las comunidades supieron reaccionar y el impulso inicial se quedó : hoy en día una dinámica nueva se ha puesto en marcha.

E1 balance provisorio de la acción del grupo "Autóctono", en un ambiete tan difícil como lo es el de La Vega, resulta se positivo - más que todo en lo que se refiere a la participación, la construcción identitaria y la cohesión de la comunidad - pero esta organización debe enfrentarse diariamente a una pobreza y una violencia crecientes.

Sin embargo, en ambos casos (Barlovento, La Vega) el trabajo socio-cultural llevado a cabo por las organizaciones populares, por más de diez años, con el apoyo de las poblaciones ha demostrado ser un camino válido por la búsqueda de una nueva modernidad integrada por una identidad (re)construida. Semejantes movimientos podrían contribuir a una nueva definición de la identidad nacional reconocedora de las diversidades regionales y étnicas ${ }^{30}$.

Gracias a estas experiencias, la cultura popular, por fin despojada de carácter folklórico, podría comprobar su capacidad de adaptación a la modernidad, modernidad que había sido por mucho tiempo confiscada por las élites. Se abren igualmentee perspectivas en la busqueda de un desarrollo sustentable, en contacto con las realidades

29. Entrevista con Jesús Garcia, Presidente de la Fundación Afroamérica, Caracas, 29/11/1996.

30. Garcia Canclini, N. (Coord.) Culturas en globalización, Ed. Nueva Sociedad, Caracas, 1996. 
de las minorías "invisibles" como lo ha sido la negra en América latina $^{31}$.

Es dentro de esta perspectiva donde se ubica la investigación que llevamos actualmente a cabo en el marco del CRPLC/CNRS, ampliando nuestro terreno de estudio a un conjunto representativo de las comunidades negras en Venezuela y deseando intercambiar datos y conclusiones con investigadores que trabajan sobre otras sociedades carbeñas.

31 . Friedeman, N. de "Estudios de negros en la antropologia colombiana » in Un siglo de investigación social : antropologia en Colombia, Ed. Etno, Bogotá, 1984. 


\section{Résumé}

Depuis quelques années, on peut observer au Venezuela un renouveau des pratiques culturelles liées à l'identité noire. Ce processus a été complexe et s'inscrit dans l'affirmation d'une nouvelle société civile indépendante du système des parties et des structures traditionnelles de pouvoir. Les organisations noires ont investi de nombreux domaines de la vie sociale: défense de l'environnement, économie, santé, éducation, culture. La mise en évidence de la relation entre lutte ethnico-culturelle et revendication sociale a été au cœur de son action. Des exemples choisis dans des communautés rurales et urbaines illustreront cette nouvelle réalité.
Resumen

Desde hace unis años se puede observar una nueva affirmación de la cultura y de la identidad afrovenezolana se trata del resultado de un proceso complejo. Su característica más revelante fue su emergencia dentro de la "nueva sociedad civil" distanciada de los partidos politicos y de los poderes tradicionales. El movimiento negro venezuelano se ha impuestio como interlocutor en varios campos de la vida pùblica defensa del medio : ambiente, economia, salud, educación, cultura. Frecuentemente su acción se ha caracterizado por la voluntad de ligar lo étnico y lo social como se podrá demostrar con ejemplos escogidos en varias comunidades urbanas y rurales.

\footnotetext{
Mots-clés _ - _ - - - - - - - - - - - $-\quad-\cdots-\cdots-\cdots-\cdots-\cdots-\cdots-$ Keys-words

Afro-americanisme - Ethnicité Société civile - Culture populaire

Afro-amercanismo - Cultura popular - Etnicidad - Sociedad civil
} 